\title{
INCLUSIÓN DE LOS INMIGRANTES EN LOS CENTROS ESCOLARES PÚBLICOS ESPAÑOLES
}

\author{
INCLUSION OF IMMIGRANTS IN PULIC \\ EDUCATION CENTRES
}

Silvia Facal Santiago*
sfacal@ude.edu.uy

\section{RESUMEN}

El siglo XX y lo que va del XXI se han caracterizado por presentar movimientos de migraciones masivas los cuales vinculan diferentes factores como el de la educación.

España ha sido, desde la década de 1990, uno de los principales destinos de olas migratorias procedentes de los más variopintos lugares. El impacto de la población extranjera en los centros escolares se ha comenzado a sentir recién a partir de mediados de la década de 2000. Debido al crecimiento constante del alumnado extranjero en los centros escolares públicos españoles el gobierno se ha marcado como reto el de la integración de los mismos a través del modelo de inclusión.

En este artículo se presentan las principales políticas de inclusión de alumnos extranjeros en los centros escolares públicos españoles a través de una metodología cuantitativa y cualitativa junto con la utilización de fuentes estadísticas secundarias y análisis de contenido.

La respuesta de las autoridades de educación, a través de diversas políticas de inclusión, ha diversificado, en los últimos años, sus esfuerzos para incluir a los alumnos extranjeros en los centros escolares públicos españoles.

Palabras clave: Inclusión; alumnado extranjero; centros escolares públicos; España

\section{ABSTRACT}

The twenty-first and twenty century so far have been characterized by presenting movements of mass migration which link different factors such as education.

Spain has been, since the 1990s, one of the main destinations of migratory movements coming from the most diverse places. The impact of the foreign population in schools has begun to feel only since mid 2000. Due to the constant growth of foreign students in Spanish public schools the Spanish government has set itself the challenge of integrating them through the inclusion model.

\footnotetext{
* Doctora en Historia de América y Contemporánea por la Universidad de Santiago de Compostela. Máster Universitario en Inmigración (Especialidad Investigación e Intervención social) por la Universidad Pontificia Comillas de Madrid. Especialista en Gestión de Empresas Turísticas por la Universidad Politécnica de Madrid. Diploma en Docencia Universitaria por la Universidad de Montevideo. Licenciada en Historia por la Universidad de Santiago de Compostela y Licenciada en Ciencias Históricas por la Universidad de la República. Está categorizada como investigadora activa nivel 1 en el Sistema Nacional de Investigadores de la ANII. Actualmente se desempeña como docente en la UCU, UDE y UM. Tiene a su cargo la línea de investigación de las migraciones de ida y vuelta en Uruguay.
} 
In this article the main inclusion policies of foreign students are presented in Spanish public schools through a quantitative and qualitative methodology and the use of secondary statistical sources and content analysis.

The response of the authorities of education has diversified in recent years, efforts to include foreign students in Spanish public schools through the inclusion model.

Keywords: Inclusion; immigrants; public education centres; Spain.

\section{INTRODUCCIÓN}

El siglo XX en su totalidad y los inicios del siglo XXI se han caracterizado no sólo por ser de los más violentos de la historia sino también por p0seer los mayores movimientos o flujos migratorios producidos por razones de diverso índole.

Los factores que han llevado a las personas a trasladarse fuera de sus fronteras durante el pasado siglo y los inicios del presente pueden ser de carácter individual o que afectan a un gran número de personas produciendo con ello las denominadas migraciones masivas. Con relación a las migraciones de carácter individual ${ }^{1}$ las principales causas que las propician suelen ser: las culturales; las educativas (fuga de cerebros); las psicológicas; los cambios en el modo de vida; la aventura... Al mismo tiempo pueden estar acompañadas también por otros motivos de orden económico, social, político, religioso, etc. Las migraciones, en cambio, de carácter masivo, suelen estar causadas por razones de orden económico, social, político, religioso, racial o étnico, medioambientales, etc. Entre los principales factores económicos de expulsión masiva encontramos, a grandes rasgos, los siguientes: nivel de renta más bajo que en destino para cada perfil socio-ocupacional, considerando la calificación, el sexo y la edad; imposibilidad total o parcial de acceder a la propiedad y usufructo de los recursos (por ejemplo: latifundio); nivel de desempleo más alto que en destino, en relación con el total de la población y con el perfil personal; crisis del sector económico al que pertenece el candidato a emigrar (por ejemplo: procesos de desindustrialización); inestabilidad económica o clima de crisis

::114:: generalizada, con impacto social; expectativas de escasa movilidad socioeconómica; alta presión demográfica: una gran cantidad de población, sobre todo la más joven, que no encuentra formas de sustento suficiente o satisfactorias; población distribuida heterogéneamente en el territorio, por lo general con procesos de gran concentración urbana en condiciones de hacinamiento; grandes niveles de pobreza e insatisfacción de las necesidades humanas básicas e intermedias a pesar de invertirse grandes horas de trabajo para ello; deterioro de los niveles de renta y de satisfacción de las necesidades humanas básicas e intermedias por la crisis de las políticas sociales como consecuencia de los Planes de Ajuste Estructural, "economías de guerra" y otras medidas de "estabilización económica"; búsqueda de mejora en la calidad de vida en el sentido, por ejemplo, de disponer de una mayor cantidad de horas para disfrutar de su tiempo libre, de su familia y del fruto de su trabajo o jubilación; otros incluso, emigran para estar con sus familiares emigrados previamente poniendo en funcionamiento las cadenas migratorias.

Se puede afirmar, de cierta manera, que las migraciones del siglo XX y principios del XXI producidas por los distintos factores señalados con anterioridad, tiene un fuerte impacto en las estructuras psicológicas, culturales y sociales de los migrantes, así como efectos también en sus estilos de vida, demandando servicios específicos de salud, educación y vivienda (Jurado de los Santos \& Ramírez Iñiguez, 2006).

En los centros de educación formal -en mayor medida los de primaria y secundaria públicos de los países receptores de los movimientos masivos migratorios se suele encontrar niños/as y adolescentes de diversas nacionalidades y grupos étnicos con culturas hasta a veces muy distantes a las de las sociedades de acogida. Estas últimas se han propuesto, a través de diversos modelos, integrar a estos estudiantes de diferentes orígenes buscando, en una primera instancia, asimilarlos y luego, de forma más reciente, incluirlos reconociendo su diversidad y apostando por una educación intercultural.

1. En este trabajo no nos centraremos en las mismas porque sus efectos se producen sobre un número reducido de migrantes. 
En este artículo presentaremos, precisamente, los modelos de inclusión de los inmigrantes en edad escolar en los centros escolares públicos españoles a los cuales concurre el $82 \%^{2}$ de los mismos (Ministerio de Educación, Cultura y Deporte, 2012), como caso paradigmático debido no sólo a la fuerte inmigración recibida por España a partir de la década de 1990 hasta la actualidad ${ }^{3}$ sino también porque el citado país se ha convertido, a partir de 2002, en el principal polo de atracción de migrantes uruguayos los hijos de los cuales concurren también a los centros educativos españoles. En sociedades como la española en las cuales los inmigrantes representan ya el $12 \%$ aproximadamente de su población total (INE, 2014) y los residentes menores de edad son el $20 \%$ aproximadamente del total de la población extranjera -en su mayoría población en edad escolar obligatoria- se hace necesario incluir de forma adecuada a estos últimos en los centros de educación.

A partir de principios de la década de los 2000 se produjo un aumento continuo en la matriculación de alumnos extranjeros en el sistema educativo español superando el $25 \%$ de crecimiento anual durante cinco años consecutivos lo cual elevó el número de escolares extranjeros desde los 80.587 que había en el curso escolar de 1998-99 a 449.936 en el curso 2004-05 (Ministerio de Educación Ciencia y Deporte, 2012). De este modo, la tasa de matriculación en los centros escolares españoles que había comenzado a disminuir a partir de la década de 1990, cayendo a una tasa del $2,5 \%$ anual (Rahona López \& Morales Sequera, 2013), ha registrado una continua reducción de las tasas de crecimiento de la población escolarizada. Como efecto de lo anterior se puede concluir que la entrada de población escolar extranjera ha producido un continuo crecimiento del porcentaje de estudiantes en las aulas. Este continuo crecimiento de alumnos extranjeros escolarizados ha llegado a propiciar que se instaure en la sociedad española la impresión generalizada de que el número de inmigrantes presentes en las aulas es muy alto (Garrido \& Cebolla, 2010); aunque esta visión es un tanto exagerada debido a que si bien el alumnado extranjero ha aumentado exponencialmente a partir del curso 2004-05 tan sólo uno de cada diez alumnos es extranjero (Ministerio de Educación, Ciencia y Deporte, 2012). No obstante, el grado de heterogeneidad de la población extranjera es muy amplio junto con su distribución entre las Comunidades Autónomas españolas que configura perfiles étnicos específicos en cada una de las mismas y también se debe tener en cuenta las importantes diferencias entre centros educativos según su titularidad que ha provocado una alta concentración de escolares en centros públicos.

\section{METODOLOGÍA Y TÉCNICAS UTILIZADAS}

Esta investigación se desarrolló a través de un enfoque metodológico mixto con un predominio del cuantitativo sobre el cualitativo debido a que se hizo necesario contar con información de carácter estadística sobre la realidad de los alumnos extranjeros en los centros públicos españoles. El enfoque cualitativo, complemento del cuantitativo, nos permitió analizar e interpretar los programas actuales de inclusión aplicados en los citados centros de educación a nivel del estado español y de las Comunidades Autónomas en particular.

Las técnicas utilizadas de la mano del enfoque cuantitativo fueron las estadísticas secundarias provenientes del Instituto Nacional de Estadística de España y del Ministerio de Educación, Ciencia y Deporte de España. Estas fuentes de información son de fácil acceso y están actualizadas hasta el año 2012, un año antes de la realización de la investigación.

En cambio, las técnicas cualitativas utilizadas en esta investigación fueron los análisis de contenidos realizados a las propuestas y programas de inclusión realizados por las Comunidades Autónomas españolas. De este modo, pudimos conocer sus implicancias y necesidades de aplicación en el nuevo contexto del aumento del alumnado extranjero en las aulas de los centros escolares públicos españoles desde el curso 2004-05.

2. Esto se puede deber a que los inmigrantes suelen escolarizar a sus hijos en centros en los cuales su minoría étnica está muy presente, como mecanismo para favorecer la integración de sus hijos en el sistema educativo formal e incluso como manera de evitar una posible discriminación por parte de la población autóctona hacia los niños recién llegados (Rahona López \& Morales Sequera, 2013).

3. A pesar de la fuerte recesión económica vivida por España a partir de 2008 a este país continúan llegando migrantes de diferentes orígenes. 


\section{LA VARIABLE EDUCACIÓN COMO ELEMENTO DE INTEGRACIÓN DE LOS MIGRANTES EN LAS SOCIEDADES RECEPTORAS}

La variable educación juega un papel preponderante en los movimientos migratorios en todas sus perspectivas:

- Funciona como una causal de nivel fundamentalmente individual de los mismos.

- Se configura como una de las variables utilizadas para medir la integración de los inmigrantes en las sociedades de acogida y se verifica de la siguiente manera: matrícula en centros educativos de todos los niveles públicos y privados -incluyen los centros de educación étnica-; ausentismo escolar; calificación y pasaje de cursos; becas.

- Tiene al mismo tiempo una incidencia tanto en las sociedades receptoras como en de las de destino estudiadas a través de la denominada "fuga de cerebros" gracias a las cuales estas últimas se ve beneficiadas. La "fuga de cerebros" se suele producir cuando en las sociedades de origen existe un acceso restringido a estudios de perfeccionamiento de posgrado o falta de investigación, principalmente.

Si los extranjeros no se escolarizan o tienen serios problemas de convivencia escolar, esto provoca una menor integración en la sociedad receptora y hasta conflictos. El encuentro entre diversas culturas suele provocar conflictos debido a la diversidad de valores y de costumbres. Sin embargo, el auténtico conflicto no viene tanto de las diferencias culturales sino de las económicas, sociales y étnicas. En este sentido, Girard \& Koch (1997) señalan que el conflicto en sí mismo no es positivo ni negativo, sino más bien una parte natural y consustancial de la vida escolar, partiendo, además, de la importancia de aprender a mirar el conflicto, a entenderlo y a analizarlo desde una perspectiva de apertura y diálogo. En este punto, debemos decir que la educación intercultural promueve relaciones de igualdad y de mutuo enriquecimiento entre personas procedentes de culturas diferentes, mediante el aprendizaje de valores, habilidades, actitudes y conocimientos interculturales; $y$ realmente tenemos que explicitar que en este marco es donde se ponen en juego las estrategias de gestión y regulación de los conflictos en los contextos educativos de diversidad.

Los mayores problemas de integración de los alumnos extranjeros se presentan en edades que oscilan los 10 a los 16 años: si hay una diferencia importante del idioma, por ejemplo, se ven incapaces de seguir las clases y suelen abandonar. Incluso se pueden producir problemas debido a la utilización de códigos de referencia cultural distintos que pueden terminar produciendo malentendidos.

Actualmente, en los centros escolares, y los españoles no están exentos de ello, se enseña en la denominada pedagogía de la indiosicracia blanca la cual enseña por igual a los blancos y a los no blancos cuáles han de ser los patrones adecuados basada en la ilustración europea (Kincheloe y Steinberg, 1999).

La escuela transmite una cultura escolar que tiene mucho que ver con la propia cultura de la sociedad en la que se encuentra, y que, en términos generales, responde a las necesidades del grupo culturalmente mayoritario (Sabariego, 2002). Y quien acoge puede terminar discriminando a quien no se comporte según los parámetros esperados. Se entiende, por lo tanto, por discriminación:

\footnotetext{
"a la imposibilidad del pleno ejercicio de los derechos y garantías de ciertos sectores sociales de la población en razón del sexo, raza, creencias religiosas o políticas, nacionalidad, situación social, elección sexual, edad y discapacidades. La discriminación se ha ejercido siempre sobre todo aquello que se aparta, aquello que es diferente de lo hegemónico ya sea en lo social, en lo político o cultural. Lo 'diferente' por razones de nacionalidad, religión, raza, elección sexual, edad etc." (Lipszyc, 2006)".
}

No se debe olvidar que la escuela actúa como dispositivo de reproducción cultural de la conducta de la sociedad en general. La discriminación, el racismo y la exclusión en las escuelas es un apéndice más de lo ocurrido en otros ámbitos macro, como puede ser la interacción en el barrio. Junto con ello transmite, según manifiesta Lipszyc (2006), un sistema de lealtades, la competencia, la creatividad, la solidaridad o su ausencia, el autoritarismo y las jerarquías como forma natural de las relaciones sociales. Al mismo tiempo, llega a difundir todo un "currículum oculto" que, en la práctica 
funciona como uno de los más importantes disciplinadores que posee un Estado. Junto con ello, la escuela puede ser también el ámbito propicio para expandir y ejercitar la antidiscriminación (Lipszyc, 2006).

Por ello, tanto para los alumnos y alumnas pertenecientes a grupos étnicos o culturales minoritarios la cultura escolar puede significar una especie de lastre que dificulta sus procesos de adaptación o integración (Essomba, 1999).

En estos contextos surgen en España diversos conflictos vinculados al multiculturalismo escolar (Bartolomé, 1997a; Jordán, 1999; Essomba, 2003):

a) Dificultades escolares. Muchos de los alumnos inmigrantes que llegan a las escuelas públicas proceden de familias humildes, con pocos recursos económicos y educativos, y algunos vienen con la experiencia de una escasa o nula escolarización, o con grandes diferencias con respecto a las que se producen en el educativo español; esto hace que algunos muestren inicialmente unas carencias importantes que pueden dificultar, en unos primeros momentos, su integración con el resto del alumnado.

b) Desconocimiento del idioma. Esto supone inicialmente una dificultad mayor para los alumnos que proceden de culturas muy diferentes en las escuelas españolas. En este sentido, en éstas el citado problema se está resolviendo mediante la puesta en marcha de las denominadas Aulas Temporales de Adaptación Lingüística (ATAL). Sin embargo, este desconocimiento no sólo tiene repercusiones iniciales en el aprendizaje y ritmo escolar de los alumnos de origen inmigrante sino también en los componentes afectivos los cuales son importantes cuando estos alumnos se sienten impotentes ante la falta de comunicación o el estado de cierto aislamiento relacional.

Para poder resolver este tipo de situaciones, la UNESCO (2003) estableció tres principios básicos que deben ser tomados en cuenta en la educación en contextos multilingües:

- Apoyar la enseñanza de la lengua materna como medio para mejorar la calidad de la educación con base en la experiencia de alumnos y profesores. Este principio se fundamenta en el supuesto de que la lengua materna es esencial en la educación inicial y en el alfabetismo, para lo que es necesaria la formación del profesorado y el material de lectura correspondiente.

- Apoyar la educación bilingüe y multilingüe en todos los niveles educativos así como promover la igualdad social y de género como elemento clave de las sociedades lingüísticamente diversas. Debe estimularse la comunicación y el desarrollo de la capacidad de diálogo primero en la lengua materna y luego en la lengua nacional oficial y/o en otras. Asimismo, el intercambio de profesores entre países posibilita el desarrollo de habilidades lingüísticas. Con respecto a la política educativa, hay que enfatizar la promoción de la enseñanza de las diferentes lenguas a través de la disposición de materiales en formato electrónico.

- Reconocer la diversidad lingüística como un componente esencial de la educación intercultural para estimular el entendimiento entre diferentes grupos de personas y asegurar el respeto de los derechos fundamentales. Este principio se basa en la afirmación de que los derechos educativos de las minorías debe ser respetada mediante la implementación del derecho de aprender la lengua materna, el derecho de aprender la lengua oficial así como las lenguas globales de comunicación. De igual modo, este principio está orientado a la valoración positiva de la diversidad cultural a través de una reforma del currículo en el que se incluya la lengua, historia, cultura e identidad de las poblaciones minoritarias.

c) Aumento excesivo de alumnos de origen inmigrante en las escuelas públicas. La llegada de inmigrantes ha supuesto que muchos de ellos se fueran a vivir a ciertos barrios de las ciudades donde el precio de las viviendas es más asequible, lo cual provoca una cierta concentración -en especie de guetos de inmigrantes- que hace que muchos de los centros educativos se nutran cada vez más de una población mayoritariamente (o casi) de origen inmigrante; esto puede suponer una suerte de "guetización" de estos centros escolares públicos. 
d) Aparición de algunos conflictos religiosos. En algunos centros escolares públicos en países en donde hay grandes diferencias religiosas entre la población migrante y la local-como el caso de Francia, por ejemplo- se vive con mayor impacto noticias, que salen en los medios de comunicación, sobre la ostentación de determinados símbolos religiosos en las aulas o simplemente por seguir conductas prescriptas en la religión practicada por ellos y sus padres. Un ejemplo de ello sería el caso del pañuelo o velo islámico, la negación por parte de algunas familias a que sus hijas no realicen determinadas actividades escolares porque contravienen normas religiosas, etc..

Actualmente, en España se están dando también estos conflictos acompañados de controversias y para tratar de resolverlos, en algunos casos, se ha tomado el ejemplo del estado francés. Se recuerda aún cuando en Francia, en 1989, se expulsó a tres chicas marroquíes de un instituto de las afueras de París por negarse a quitarse el pañuelo de la cabeza en clase. El debate polarizó Francia y se acordó tratar este asunto caso por caso. El Tribunal Supremo francés se limitó a prohibir cualquier vestimenta "ostensible" o "provocadora" que perturbara el orden del programa educativo. Un caso similar ocurrió en 2007 en España, concretamente en Girona: en una escuela pública de la citada localidad se prohibió a una niña a concurrir a clase porque llevaba un velo islámico. Como respuesta al incidente, el Departamento de Educación decidió obligar a la escuela Anexa de Girona a admitir a la niña de 6 años que hacía una semana no asistía a la escuela porque el centro le había comunicado a los padres que no podía usar el velo en clase. El centro, para ello, se acogía a su reglamento interno, que rechazaba cualquier diferencia entre los alumnos por razones de sexo, religión u otros factores. No obstante, el director de los servicios territoriales de Educación afirmó que no había ninguna razón la cual pudiera justificar el absentismo de la niña, que podría "volver a la escuela mañana mismo" (La Vanguardia. 1-10-2007).

e) Escasa implicación familiar en las tareas escolares. La investigación realizada por Rascón (2006) -sobre inmigrantes marroquíes en España- nos lleva a tener en cuenta que muchas familias de origen inmigrante (en este caso, magrebí) no ayudan a sus hijos a realizar las tareas escolares domiciliarias, debido principalmente al desconocimiento o a sus obligaciones laborales las cuales les hacen pasar mucho tiempo fuera de casa. No obstante, esta misma investigación muestra la

::118:: satisfacción de los padres marroquíes, a modo de ejemplo, por la educación recibida por sus hijos en las escuelas públicas españolas, así como las altas expectativas sobre las oportunidades que el propio sistema educativo pueda proporcionarles a sus hijos.

\subsection{Hacia la inclusión de los niños extranjeros en los centros escolares españoles}

Es una realidad, como ya se ha manifestado, que a partir del curso escolar 2004-05, los centros escolares públicos españoles acogen a un mayor número de alumnos extranjeros y que su distribución supone diferencias entre las comunidades autónomas. Inclusive, el origen de los mismos es muy heterogéneo. El gobierno español, en general, y las Comunidades Autónomas, en particular, se han marcado como reto integrar a estos alumnos apostando por la "inclusión" de los mismos.

El término inclusión - que debe reconocer la diversidad- en educación se ha definido de diversas maneras. A continuación se pueden leer una serie de definiciones sobre inclusión; todas ellas tienen en común el hecho de integrar a todos los alumnos en los centros educativos eliminando, para ello, las barreras limitantes:

- "La inclusión no tiene que ver sólo con el acceso de los alumnos y alumnas con discapacidad a las escuelas comunes, sino con eliminar o minimizar las barreras que limitan el aprendizaje y la participación de todo el alumnado" (Blanco 2002: 6).

- "La inclusión se concibe como un conjunto de procesos orientados a eliminar o minimizar las barreras que limitan el aprendizaje y la participación de todo el alumnado" (Booth y Ainscow 2002: 9).

- "Inclusión como una aproximación dinámica que responde positivamente a la diversidad de los alumnos y ve las diferencias no como un problema sino como oportunidades para enriquecer" (UNESCO 2005: 12-13). 
- "La inclusión social es un derecho natural del sujeto que proviene de su condición gregaria; como derecho humano debe estar garantizado" (Rivas 2006: 365).

- Las formas de pensar la inclusión teniendo en cuenta a la Declaración de Salamanca en 1994, serían según Ainscow y César (2006): 1. la que está relacionada con la discapacidad y las necesidades educativas especiales y que tiene como finalidad fomentar la participación de los alumnos que tienen alguna discapacidad física o intelectual; 2. la inclusión como respuesta a la exclusión de aquellos alumnos que son categorizados como de mala conducta; 3 . la inclusión de grupos vulnerables que no tienen acceso a la escuela por razones de pobreza o discriminación; 4. inclusión vista como una escuela común con estrategias de enseñanza y aprendizaje inclusivas; 5 . inclusión como educación para todos en el que se enfatiza una educación y una escolarización sin excluir a nadie por razones físicas, intelectuales, económicas o culturales.

Por tal motivo y como lo establece la Declaración de Salamanca (1994:7):

"[...] las escuelas deben acoger a todos los niños, independientemente de sus condiciones físicas, intelectuales, sociales, emocionales, lingüísticas u otras [...] la experiencia nos ha demostrado que se puede reducir el número de fracasos y de repetidores [y que] puede servir para evitar el desperdicio de recursos y la destrucción de esperanzas, consecuencias frecuentes de la mala calidad de la enseñanza y de la mentalidad de que "la que sirve para uno sirve para todos"'.

Para el logro de la deseada inclusión en las escuelas públicas españolas se deberían tener en cuenta una serie de dimensiones, de la escuela inclusiva (Booth y Ainscow, 2002):

- La cultura: una cultura inclusiva implica la creación de una comunidad escolar segura, acogedora, colaboradora donde cada alumno es valorado.

- Las políticas: a partir de una cultura escolar inclusiva se crean políticas en las que se determinan los lineamientos dirigidos a dar respuesta a la diversidad del alumnado y a mejorar su aprendizaje y su participación.

- Las prácticas: en las mismas se asegura que las actividades en el aula y fuera de ella promuevan la participación del alumnado y se tenga en cuenta su experiencia y conocimiento adquirido tanto en la escuela como en otros ámbitos.

De acuerdo con la UNESCO (2005) los componentes clave en una escuela caracterizada por ser inclusiva son:

- La política educativa

- Los profesores

- Los padres

- El currículum

- Las estrategias de enseñanza

- Los agentes que apoyan indirectamente la educación de los alumnos, como: los institutos de capacitación del personal y del profesorado

Es clave también, el logro de una adecuada interculturalidad4; además del reconocimiento y la adaptación de las diferentes culturas se propone un diálogo entre ellas, en condiciones de igualdad y reciprocidad, que permitan la convivencia. 
La interculturalidad cumple una función clave, entendiendo que no sólo es suficiente la aceptación y tolerancia de alumnos de diversas culturas en un centro escolar, sino también es necesario su propio reconocimiento y efectiva atención desde los diferentes componentes de la educación, desde el currículum y sus elementos hasta los procesos de enseñanza aprendizaje en el aula (Jurado de los Santos y Ramírez Iñiguez, 2010). Siguiendo con esta tónica, Sánchez (2004) distingue cuatro modos de relación de las instituciones escolares en las realidades multiculturales con las que trabaja -la última de ellas se identifica con la inclusión-. Dichas relaciones son:

- De asimilación, la cual se basa en los principios de la cultura mayoritaria que incorpora la cultura de procedencia a sus prácticas educativas sin adaptaciones y sin tomar en cuenta otras aportaciones.

- De diferenciación, en la cual las instituciones tratan de dar respuesta a las características culturales de los grupos minoritarios ofreciendo posibilidades paralelas de escolarización. Estos grupos son reconocidos, cada uno en su barrio y en su escuela pero sin interacción entre ellos.

- De integración, donde además del reconocimiento de las minorías culturales se reconocen sus derechos culturales y educativos asumiendo un trato igual para todos los alumnos sin hacer distinciones por su procedencia social o pertenencia a determinados grupos étnicos o culturales. En este sentido, todos asisten a las mismas escuelas con los mismos profesores.

De esta forma, la escuela debe procurar la interculturalidad evitando el conflicto entre los hábitos, las costumbres y las actitudes de los alumnos, por lo que la comunicación entre la comunidad escolar y los agentes involucrados en la educación de los alumnos migrantes -padres, profesores, administradores, trabajadores sociales, orientadores- es vital.

Chieh y Vázquez (2009) recomiendan siete acciones que la escuela debe emprender para favorecer

::120:: la inclusión de los alumnos en contextos multiculturales como el de las escuelas públicas españolas. Éstas son:

- Desarrollo de un equipo profesional encargado de incrementar la competencia intercultural entre los estudiantes;

- Aprendizaje de las culturas de los estudiantes a través del contacto directo con las familias y de actividades multiculturales;

- Enseñanza del idioma del lugar de acogida para los padres y del idioma de los niños para los profesores y el personal de la escuela;

- Incluir en el currículo la enseñanza de la cultura y de la historia de los lugares de procedencia de los niños migrantes;

- Realización de talleres y portafolios sobre las diversas culturas presentes en la escuela;

- Trabajar con grupos de apoyo a los padres;

- Realización de talleres informativos sobre el sistema educativo y aspectos legales que los padres deben saber.

La inclusión de los alumnos extranjeros en los centros escolares públicos españoles a través de los mecanismos anteriores se logra principalmente en el ámbito de la escuela pública la cual funciona también como formadora de nuevos ciudadanos y como un espacio integrador de los niños provenientes de diferentes culturas. 


\subsection{Principales programas de inclusión en España}

Entre los principales programas que las Comunidades Autónomas españolas han puesto en funcionamiento para favorecer la integración y la inclusión de los alumnos extranjeros en los centros escolares públicos españoles, en el marco de las políticas del gobierno central, tenemos:

- Andalucía: Plan para la Atención Educativa del Alumnado Inmigrante (2001-04).

- Canarias: Plan Sur (2002-06).

- Castilla y León: Plan Marco de Atención Educativa a la Diversidad (2004-06).

- Murcia: Plan Regional de Solidaridad en Educación (2001-03).

- País Vasco: Programa para la atención del alumnado inmigrante (2004-06).

Los planes anteriores tienen en común el hecho de buscar la integración de los alumnos extranjeros en las aulas escolares de los centros públicos a través de su inclusión en las mismas a través de diversas estrategias.

Al mismo tiempo, la mayoría de las Comunidades Autónomas han puesto en funcionamiento también programas específicos de acogida dirigidos a los alumnos extranjeros. Según Martínez de Lizarrondo (2009) las Comunidades Autónomas que más han profundizado en los mismos son: Aragón, Baleares, Canarias, Navarra y el País Vasco.

Junto con la anterior las Comunidades Autónomas cuentan con profesionales especializados en la tarea de acogida de los alumnos extranjeros. Estos profesionales son profesores tutores o mediadores que prestan su apoyo en las denominadas "aulas de acogida".

Incluso, todas las Comunidades Autónomas españolas conscientes de la importancia de la implementación de medidas que tengan en cuenta los orígenes diversos de los alumnos de los centros escolares públicos españoles han incorporado el plan de atención a la diversidad en cada una de ellas.

No obstante, la inclusión de los inmigrantes en los programas de compensación educativa es una medida no exenta de cierta polémica. Al principio, la compensación se dirige principalmente a los alumnos con deficientes condiciones de escolarización y/o lingüísticas -no sólo de la lengua española sino también de las lenguas propias de algunas Comunidades Autónomas como el catalán, el gallego, el vasco, el valenciano o el balear-. Estos programas, que consisten en determinadas materias y terapias pedagógicas, poseen un elemento diferenciador, y se realizan fuera del aula de clase. La Comunidad Autónoma que más ha trabajado al respecto es la de Madrid (Rahona López \& Morales Sequera, 2013).

Las Comunidades Autónomas españolas también han puesto en funcionamiento programas relacionados con la atención a las familias de los alumnos extranjeros. El entorno de los alumnos es, como ya hemos comentado, un indicador fundamental de su integración en las aulas de clase públicas españolas. Por tal motivo es necesario poner en marcha determinadas medidas dirigidas al apoyo de las familias de los propios alumnos extranjeros. Estas pueden ser de dos tipos: las destinadas a facilitar el acceso al sistema educativo y las que se dirigen a fomentar la integración y participación de las familias en los centros escolares públicos españoles a los cuales concurren sus hijos. Entre las primeras destacan medidas que favorecen el acceso de las familias al sistema educativo a través de la información que se proporciona en las comisiones locales de escolarización, de guías elaboradas con este propósito o en reuniones informativas realizadas en los propios centros educativos. Para poder facilitar esta tarea Comunidades Autónomas como las de Andalucía, Aragón, Baleares, Cataluña, Extremadura, Galicia, la Comunidad de Madrid, Murcia y la Comunidad Valenciana cuentan con un servicio de intérpretes y de mediadores culturales el cual permite mejorar la atención del alumnado extranjero. 
Junto con las medidas anteriores, las Comunidades Autónomas españolas han optado también por formar a profesores de forma adecuada para trabajar con los alumnos extranjeros. De este modo, se han puesto en marcha una serie de medidas destinadas no solo a la formación inicial sino también a la formación permanente del profesorado para brindarle estrategias y recursos que le permitan dar una respuesta educativa adecuada a las necesidades de los alumnos extranjeros. Esta formación se lleva a cabo a través de diversas modalidades como: formación en el centro; cursos; seminarios; grupos de trabajo; cursos de posgrado; etc. (Rahona López \& Morales Sequera, 2013).

\section{CONCLUSIONES}

En este artículo se ha logrado observar como la mayoría del alumnado extranjero de España se distribuye en centros escolares públicos debido a, como ya se puso de manifiesto, que los inmigrantes suelen escolarizar a sus hijos en centros en los cuales su minoría étnica está muy presente, como mecanismo para favorecer su integración en el sistema educativo formal e incluso como manera de evitar una posible discriminación por parte de la población autóctona hacia los niños recién llegados.

Al mismo tiempo se ha logrado comprobar como las autoridades españolas con injerencia en temas de educación, tanto del gobierno central como de las Comunidades Autónomas, se han trazado como política educativas prioritarias, con relación a los estudiantes extranjeros, el de su integración a través del modelo de la escuela inclusiva.

Junto con lo anterior, en el presente artículo, se han presentado los principales modelos de inclusión de los inmigrantes en edad escolar en los centros públicos españoles a través de las políticas que, en el ámbito educativo, se han puesto en marcha para facilitar la integración de los citados alumnos en el sistema educativos de un país con un porcentaje de población extranjera de un $12 \%$ aproximadamente.

\section{ÍNDICE DE FUENTES}

\section{SECUNDARIAS}

\section{Estadísticas}

Anuario Estadístico (2012). Las cifras de la Educación en España [en línea] Ministerio de Educación, Cultura y Deporte de España [fecha de consulta: 14 de diciembre de 2013]. Disponible en: http:// www.mecd.gob.es/servicios-al-ciudadano-mecd/estadisticas/educacion/indicadores-publicacionessintesis/cifras-educacion-espana.html

Padrón Municipal [en línea] Instituto Nacional de Estadística español [fecha de consulta: 23 septiembre 2014). Disponible en: http://www.ine.es/inebmenu/mnu_padron.htm

\section{Bibliografía}

AINSCOW, M. y CÉSAR, M. (2006): "Inclusive education ten years after Salamanca: Setting the agenda". En: European Journal of Psychology of Education, vol. xxi (3), pp. 231-238.

BARTOLOMÉ, M. (1997a): Diagnóstico a la escuela multicultural. Barcelona: Cedecs.

BLANCO, M. R. (2002): Ámbitos más relevantes para la integración: la escuela, Madrid, Universidad Pontificia Comillas de Madrid.

BOOTH, T. \& AINSCOW. M. (2002): Index for inclusion (2nd ED). Developing leaning and participation in schools ( $2^{\mathrm{a} e d}$ ). Manchester: CSIE [trad. Guía para la evaluación y mejora de la educación inclusiva. Madrid: Consorcio Universitario para la Educación Inclusiva.

CHIEH, L. \& VÁZQUEZ, E. (2009): "Schools consultants as agents of social justice for multicultural children and families". En: Journal of Educational and Psychological Consultation, vol. 19 (1), pp. 26-44. 
ESSOMBA, M. A. (1999): Construir la escuela intercultural. Reflexiones y propuestas para trabajar la diversidad étnica y cultural. Barcelona: Editorial Grao.

ESSOMBA, M.A. (2003). Educación e inclusión social de inmigrados y minorías. Tejer redes de sentido compartido. Barcelona. Cisspraxis.

JORDÁN, J.A. (1999). "El profesorado ante la educación intercultural”. En ESSOMBA, M.A. (Coord). Construir la escuela intercultural. Reflexiones y propuestas para trabajar la diversidad étnica y cultural. Barcelona: Editorial Grao. pp. 65-73.

JURADO DE LOS SANTOS, P. y RAMÍREZ IÑIGUEZ, A. A. (2010): "Educación inclusiva e interculturalidad en contextos de migración". En: Revista Latinoamericana de Educación Inclusiva, pp. 109-14.

GARRIDO, L. \& CEBOLLA, H. (2010): "Rendimiento educativo y concentración de inmigrantes en las escuelas españolas”. En: PISA 2006, Presupuesto y Gasto Público 61/2010, pp. 159-179.

GIRARD, K. \& KOCH, S. (1997): Resolución de conflictos en las escuelas. Barcelona: Ediciones Paidós Ibérica.

KINCHELOE, J. L. \& STEINBERG, S. R. (1999): Repensar el multiculturalismo. Barcelona: Octaedro.

LYPSZYC, C. (2006): La discriminación en la escuela. Los iguales y los otros. [en línea] [fecha de consulta: 18 de febrero de 2014]. Disponible en: http://www.revistafuturos.info/futuros14/ discriminacion_escuela.htm.

MARTÍNEZ DE LIZARRONDO, A. (2009): "Políticas autonómicas de integración de inmigrantes: la educación". En: Revista Española de Educación Comparada [en línea] Número 15 [fecha de consulta: 15 de noviembre de 2013]. Disponible en: http://revistas.uned.es/index.php/REEC/article/ view/7510

RAHONA LÓPEZ, M. \& MORALES SEQUERA, S. (2013): Educación e inmigración en España: desafíos y oportunidades. Madrid: Fundación Alternativas.

RASCON, M. T. (2006): La construcción de la identidad cultural desde una perspectiva de género: el caso de las mujeres marroquíes. Tesis doctoral. Dpto. Teoría e Historia de la Educación de la Universidad de Málaga.

SABARIEGO, M. (2002): La educación intercultural ante los retos del siglo XXI. Bilbao: Desclée.

SÁNCHEZ, S. (2004). "Respuestas educativas a los fenómenos migratorios" en: Cuevas, M. et al. ::123:: (Coord.). Atención a la diversidad y calidad educativa. Ceuta: Grupo Editorial Universitario.

UNITED NATIONS EDUCATIONAL, SCIENTIFIC AND CULTURAL ORGANIZATION (2003). Education in a multilingual world. Paris: UNESCO.

UNITED NATIONS EDUCATIONAL, SCIENTIFIC AND CULTURAL ORGANIZATION (2005). Guidelines for inclusion: ensuring access to education for all. Paris: UNESCO.

\section{Hemeroteca}

La Vanguardia (1-10-2007): Educación obliga a una escuela de Girona a admitir a una niña a quien no permitían ir a clase con velo. - See more at: http://www.acfilosofia.org/debates-27454/147-elvelo-islamico-en-los-centros-escolares\#sthash.ZDOO7tAc.dpuf 